Please do not remove this page

RMIT

UNIVERSITY

\title{
Robust methodology for fractal analysis of the retinal vasculature
}

Che Azemin, Mohd Zulfaezal; Kumar, Dinesh; Wong, T; Kawasaki, R; Mitchell, P; Wang, J

https://researchrepository.rmit.edu.au/esploro/outputs/9921861502301341/filesAndLinks?institution=61 RMIT_INST\&index=null

Che Azemin, M. Z., Kumar, D., Wong, T., Kawasaki, R., Mitchell, P., \& Wang, J. (2010). Robust methodology for fractal analysis of the retinal vasculature. IEEE Transactions on Medical Imaging, 30(2), 243-250.

https://doi.org/10.1109/TMI.2010.2076322

Document Version: Accepted Manuscript

Published Version: https://doi.org/10.1109/TMI.2010.2076322

Repository homepage: https://researchrepository.rmit.edu.au

(C) 2010 IEEE

Downloaded On 2023/04/26 19:59:06 +1000

Please do not remove this page 
Citation:

Che Azemin, M, Kumar, D, Wong, T, Kawasaki, R, Mitchell, P and Wang, J 2010, 'Robust methodology for fractal analysis of the retinal vasculature', IEEE transactions on Medical Imaging, vol. 30, no. 2, pp. 243-250.

\title{
Robust Methodology for Fractal Analysis of the Retinal Vasculature
}

\author{
M. Z. Che Azemin, D. K. Kumar, T. Y. Wong, R. Kawasaki, P. Mitchell, and J.J. Wang.
}

( 2010 IEEE. Personal use of this material is permitted. Permission from IEEE must be obtained for all other users, including reprinting/ republishing this material for advertising or promotional purposes, creating new collective works for resale or redistribution to servers or lists, or reuse of any copyrighted components of this work in other works.

Abstract - We have developed a robust method to perform retinal vascular fractal analysis from digital retina images. The technique preprocesses the green channel retina images with Gabor wavelet transforms to enhance the retinal images. Fourier Fractal dimension is computed on these preprocessed images and does not require any segmentation of the vessels. This novel technique requires human input only at a single step; the allocation of the optic disk centre. We have tested this technique on 380 retina images from healthy individuals aged $50+$ years, randomly selected from the Blue Mountains Eye Study population. To assess its reliability in assessing retinal vascular fractals from different allocation of optic centre, we performed pair-wise Pearson correlation between the fractal dimension estimates with 100 simulated region of interest for each of the $\mathbf{3 8 0}$ images. There was Gaussian distribution variation in the optic centre allocation in each simulation. The resulting mean correlation coefficient (standard deviation) was $0.93(0.005)$. The repeatability of this method was found to be better than the earlier box-counting method. Using this method to assess retinal vascular fractals, we have also confirmed a reduction in the retinal vasculature complexity with aging, consistent with observations from other human organ systems.

Index Terms-Blue Mountains Eye Study, Eye Fundus, Fourier Fractal Dimension, Gabor Wavelet

\section{INTRODUCTION}

$\mathrm{T}$ $\mathrm{HE}$ retinal vasculature is a non-invasively observable circulatory system in the eye [1-4], which provides useful

Manuscript received July 19, 2010; revised August 30, 2010. Asterisk indicates corresponding author.

*M. Z. Che Azemin is with the RMIT University, Victoria 3001, Australia, on leave from Biomedical Science Department, Kulliyyah of Science, International Islamic University, Malaysia (e-mail: zulfaezal@ieee.org).

D. K. Kumar is with the RMIT University, Victoria 3001, Australia.

T. Y. Wong is with the Centre for Eye Research Australia, University of Melbourne, Royal Victorian Eye and Ear Hospital, Melbourne, Australia, and the Singapore Eye Research Institute, National University of Singapore, Singapore

R. Kawasaki is with the Centre for Eye Research Australia, University of Melbourne, Royal Victorian Eye and Ear Hospital, Melbourne, Australia.

P. Mitchell is with the Centre for Vision Research, University of Sydney, Westmead Millennium Institute, Sydney, Australia.

J.J. Wang is with the Centre for Eye Research Australia, University of Melbourne, Royal Victorian Eye and Ear Hospital, Melbourne, Australia, and the Centre for Vision Research, University of Sydney, Westmead Millennium Institute, Sydney, Australia.

Copyright (c) 2010 IEEE. Personal use of this material is permitted. However, permission to use this material for any other purposes must be obtained from the IEEE by sending a request to pubs-permissions@iee.org." information about the microcirculation in the body. It has been found to assist in identifying health or disease in the retina and other vascular systems [5-9]. Previous studies have identified a number of features of the images of the retinal vessels that can indicate different disease processes. For example, retinal vessel caliber may provide an early indicator of the risk of coronary heart disease [8]. Other measures of retinal vascular characteristics, such as vessel tortuosity and bifurcation angle, have also been explored [10, 11].

Recent studies have attempted to summarize the structure of the retinal vasculature by fractal dimension $(F D)$, assessed using the box-counting technique, as a measure of "complexity" in a system [12-16]. These studies have demonstrated a number of possible applications of retinal vessel images. However, box-counting requires the segmentation of the vessels in the retina images. While automatic segmentation of the vessels has been developed [17], these have not been found suitable for images where there are number of distracters and there is large variation. Often there is the need for manual and time-consuming tracing of the blood vessels, and this has restricted the analysis to relatively small datasets [12-15]. Even with the semi-automated approach introduced recently, manual deletion of distracters such as light artifacts, shadows and background noise is still required [16]. Besides the cost, both the manual and automatic vessel segmentation processes are prone to error because of differences between experts making the outcome dependent on the experience of the grader, in the case of the manual process, or of variations in image color, brightness and contrast, in the case of automatic processes. There is a current need of developing a technique that could reliably measure the fractal properties of retina images without requiring segmentation methods to identify retinal vessels.

We have proposed the use of the Fourier Fractal dimension $(F F D)$ approach to compute the $F D$ of the retinal images. FFD has been used to quantify the grayscale images projected on to three-dimensional fractal surface [18]. The advantage of $F F D$ is that it computes the fractal dimension of gray scale images, and eliminates the need for image segmentation [18]. It has also been found to be relatively insensitive to noise and it is believed to work effectively with data having low signal-tonoise ratio [18, 19]. FFD has successfully been used for computing $F D$ in some biomedical applications such as for the 
analysis of the hepatic sinusoidal network in rat liver [20].

Another major difficulty associated with the analysis of retina images is the quality of the image and the need for appropriate image enhancement before $F D$ analysis. While a number of image enhancement techniques are available, wavelet analysis has been considered most suitable [21, 22]. However, wavelet transform based techniques involve selection of the most appropriate mothers of wavelet functions and scales. Soares et al. established that the Gabor wavelet is the most suitable wavelet function for retina image enhancement [22]. The most suitable scale for the FFD analysis, however, has yet not been established. We studied the impact of wavelet scales on outcomes and identified the most suitable scale for preprocessing of retina images for the fractal surface analysis.

In this manuscript, we present a methodology for obtaining the $F D$ of retinal images. This includes the image enhancement using wavelet transforms, the implementation of FFD computation, testing the robustness (repeatability and reliability) of the technique and validating the results by evaluating its associations with age, a well known association found with $F D$ in biological organs and systems [23-25].

\section{Methodology}

\section{A. Materials and Pre-processing}

We used the data collected during the Blue Mountains Eye Study (BMES), a population-based cohort study of eye diseases and other health outcomes in a suburban Australian population (age range, 50-89 years) [1, 26]. After pupil dilation, 30-degree field retinal photographs were taken using a Zeiss FF3 fundus camera. Optic disk-centred images were then digitized using a Canon CanoScan FS2710 slide scanner (Canon Corporation, Tokyo, Japan), with 24-bit colour and a maximum resolution of 2720 dpi without enhancement.

Only the healthy subjects' data were analysed. These were subjects without any known eye disease, cardiovascular disease or diabetes. This data was subdivided based on age and into 2 age groups: (1) 50-69 years; (2) 70-89 years. Random samples of right eye retina images were taken from each of these subgroups, with 193 images from the first group (50-69 years), and 187 images from the second group (70-89 years).

As a first step, these images were down sampled to $778 \mathrm{x}$ 519 pixels. This was done to reduce the computational complexity and make the resolution comparable with other similar studies [21, 22]. Only inverted green channel of the retina image were used for the analysis [22, 27, 28].

The next step was the manual identification of optic disk centre (ODC). The region of interest (ROI) was obtained by taking 150-pixel radius from the centre of the optic disk manually identified, and selected for best visibility of the vessels. The image was cropped using a bounding rectangle of size 300 x 300 pixels. The images were cropped to avoid analyzing unnecessary portion of the image.

\section{B. Vessel Enhancement}

The next step was to ensure that the analysis were not degraded by the influence of illumination, ocular media opacity and reduced contrast. The images were enhanced and represented as a three-dimensional surface using a wavelet transform, based on methods reported previously [22, 27, 28]. This was done using directional matched filter with Gabor wavelet as the kernel. Matched filter at a single scale was employed by maximizing the responses over a discrete set of kernel direction at every pixel using two-dimensional Gabor kernel for each direction.

Gabor wavelet is defined as,

$$
\psi(x)=e^{i k_{0} x} e^{-\frac{1}{2}|A x|^{2}},
$$

where

$$
A=\operatorname{diag}\left[\varepsilon^{-\frac{1}{2}}, 1\right], \varepsilon>1
$$

is a $2 \times 2$ diagonal matrix that characterizes its elongation in any intended direction. Based on the work of [22], elongation $\varepsilon$, was fixed at 4 and the vector of the horizontal and vertical frequencies, $k_{0}$ was fixed to $k_{0}=[0,3]$. The image was decomposed using wavelet transform at five different scales with $a=4,5,6,7,8$.

Maximum responses were obtained over 18 equally separated angles starting from 0 degree to 170 degrees. An example of this analysis is shown in Fig. 1 which illustrates the original image, the Gabor wavelet at 0 degree orientation, and the surface of the enhanced vessels.

\section{Fourier Fractal Dimension (FFD)}

The first step in the computation of FFD is the generation of Fourier transform of the enhanced image. The Fourier transform of the image $f(x, y)$ of size $N \times N$ is calculated as

$$
F(k, l)=\frac{1}{N^{2}} \sum \sum f(x, y) e^{-i 2 \pi\left(\frac{k a}{N}+\frac{l b}{N}\right)},
$$

where $F(k, l)$ is the Fourier transform, with $k$ and $l$ varying from $1 \ldots N$. The magnitude of the Fourier transform $(M)$ of the image is given by

$$
M(k, l)=\log \left(|F(k, l)|^{2}+10^{-6}\right)
$$




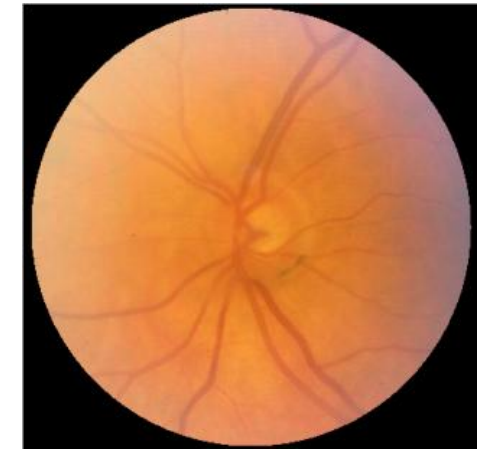

(a)

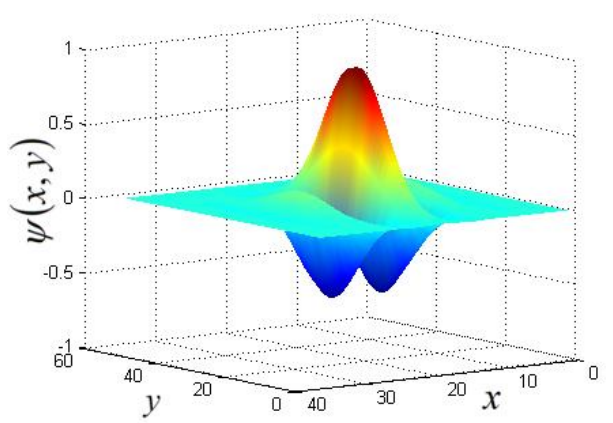

(b)

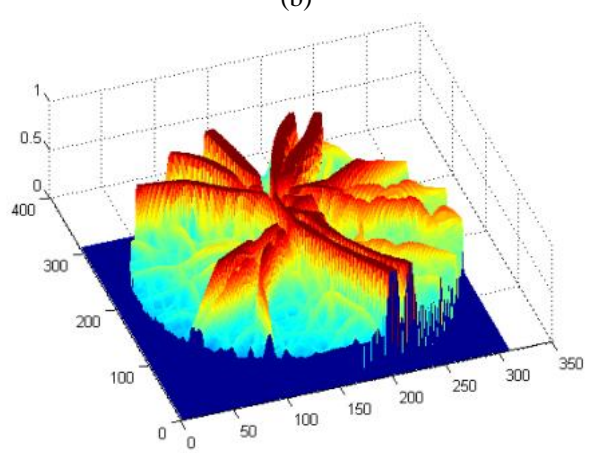

(c)

Fig. 1. Steps for vessel enhancement. (a) Original retina image cropped at 200pixel radius from optic disk center. (b) Gabor wavelet at 0 degree orientation. (c) Surface of the maximum responses of the Gabor wavelet transform over 18 angles.

where $F$ has to be radially sampled from the center of the image $[18,20]$. To obtain the overall spectrum of the image, the overall frequency, $\omega$, is the radial distance, and the corresponding magnitude of the Fourier Transform corresponding to $\omega$ is the value of $M(k, l)$ on the circle of radius $\omega$. To obtain the $F F D$, the relationship between $M$ and the $\omega$ has to be obtained on a logarithmic scale. $M$ incorporating the logarithmic function (equation 6), we plotted $M$ vs. $\log (\omega)$ as shown in Fig. 2. The FFD is related to the slope of the plot, $\beta$ as

$$
F F D=\frac{(6+\beta)}{2}
$$

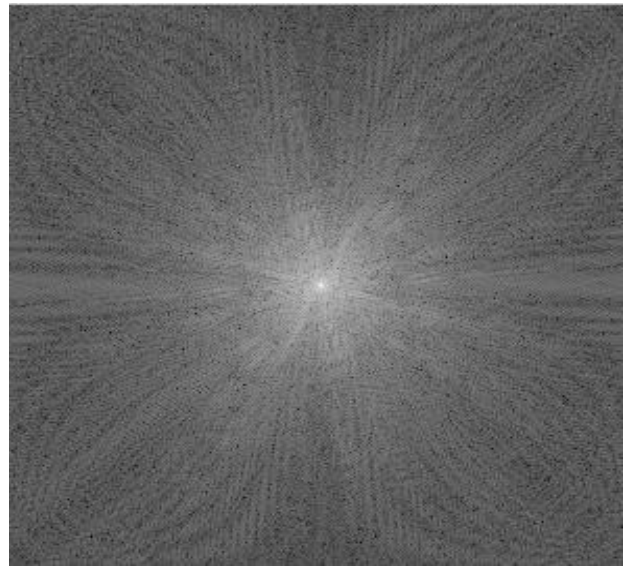

(a)

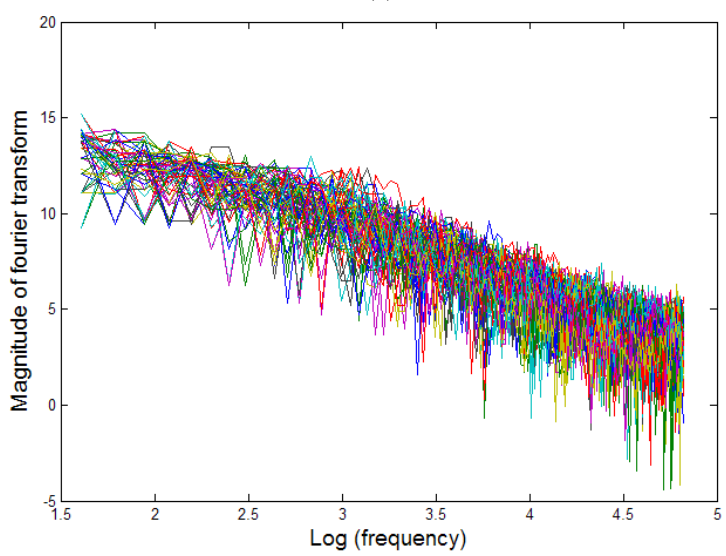

(b)

Fig. 2. Calculating the Fourier Fractal dimension. (a) Fourier transform of the retina image. (b) The slope of the Magnitude vs. $\log$ (frequency) plot, $\beta$ is associated with Fourier Fractal dimension by $F F D=(6+\beta) / 2$.

\section{Effect with Noisy Images (Robustness Test)}

$F F D$ robustness against noise was tested on the image with known FFD. This image was added with noise models typically found in biomedical images; Gaussian, Poisson, and Salt and Pepper [29]. As the first step of generating noisy images, 100 synthetic images of known FFD were generated. These synthetic images were based on the following relationship between spectral density $S(f)$ and frequency $f$,

$$
S(f) \propto f^{\beta}
$$

The values of $\beta$ corresponding to the spectral distribution of random white noise, pink noise and Brownian noise are 0 , 1 and -2 respectively. White noise comprises of spatially independent amplitudes, pink noise exists in wide range of natural phenomena and brown noise matches a non-fractal deterministic Euclidean surface.

The generation of these synthetic images follows the algorithm described by [30] which is defined as 


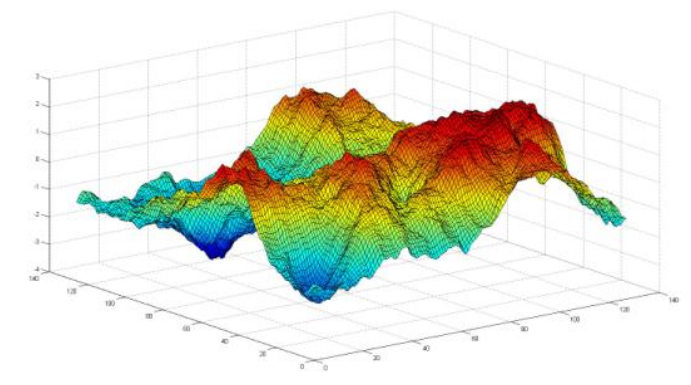

(a)

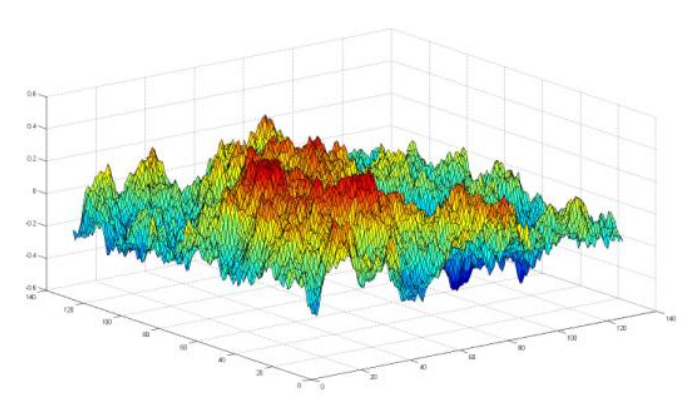

(b)

Fig. 3. Synthetic fractal images that follow $S(f) \propto f^{\beta}$ relationship. (a) Fractal dimension of 1.1. (b) Fractal dimension of 1.5. Notice that FFD increases with "coarseness".

$$
Z_{x, y}=\sum_{u=0}^{m-1} \sum_{v=0}^{n-1} \sin \left(2 \pi f_{u, v}-\theta_{u, v}\right) S\left(f_{u, v}\right)^{1 / 2}
$$

where $Z$ is an image of size $m \times n$ pixels with spatial coordinates $x$ and $y$ and $S\left(f_{u, v}\right)$ is the spectral density of frequency $f$ and the subscripts $u$ and $v$ are the frequency components in the $x$ and $y$ directions. $\theta$ is the randomizing element which is chosen independently from a uniform distribution of angles $0 \ldots 2 \pi$. The fractal dimension $F D$ is related to $\beta$ as described in (7). Fig. 3 illustrates the images generated through this process.

\section{E. Effect of Different Allocation of ROI (Repeatability Test)}

The identification of the optic centre of the image lacks precision and is often dependent on the expertise of the grader and may affect the repeatability of the outcome. To test the impact of the variation in the identification of the optic centre, and test the repeatability of the technique and its independence from the expertise of the graders, Monte Carlo simulation, based on experimentally determined variations between different graders and different images, was used to obtain 100 different optic centers and the resultant 100 different region of interest (ROI). The error of locating the ROI was assumed to be Gaussian which was also experimentally confirmed (Fig. 4).

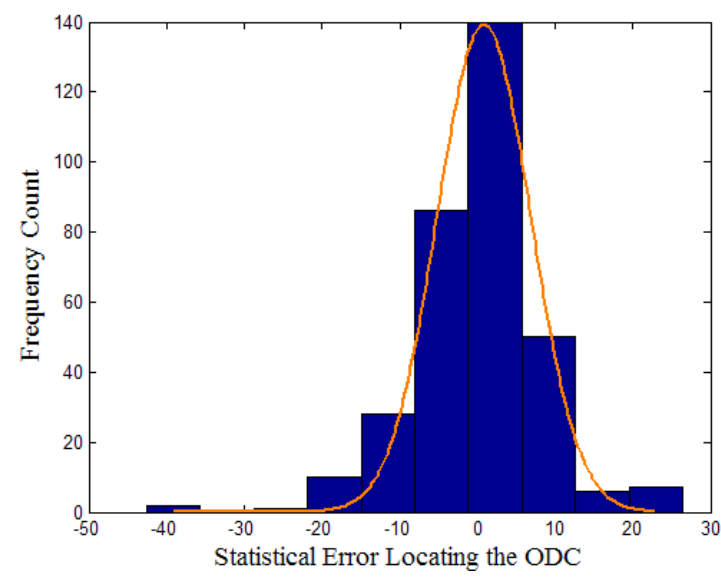

Fig. 4. Histogram describes the error distribution of locating the ODC. The line represents Gaussian fitting of the distribution.

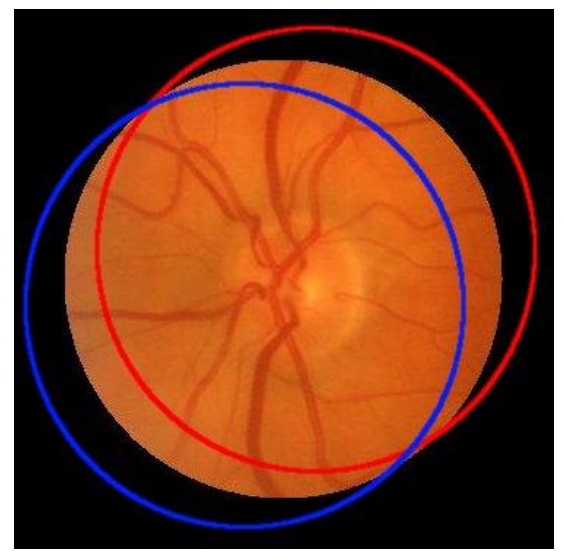

Fig. 5. Illustration of three different ROIs generated by Monte-Carlo Simulation. Each circle corresponds to one ROI.

To model the error between different graders, 15 nonexperts were asked to locate the optic disk centre (ODC) from retina images after they had been given the definition of optic disk to be an elliptical area which is brighter than its surrounding and the ODC as a convergence point to the blood vessels. They were also given one example of the ODC.

Based on the outcomes of the identification of the optic centre by the 15 lay persons, statistical error was computed

$$
\varepsilon_{i j}=X_{i j}-\mu_{j} \text { where } X=\{x, y\}
$$

where $X$ represents the $(\mathrm{x}, \mathrm{y})$ coordinate of the ODC specified by the non-expert grader, $\mathrm{i}, \mu$ is the mean location of ODC for image $\mathrm{j}$ and $\varepsilon_{i j}$ is the error which is the Euclidean distance between the mean location of ODC and the ODC identified by grader $\mathrm{i}$ of image $\mathrm{j}$. The histogram of $\varepsilon_{i j}$ for the resulting 330 error distribution populated from the 15 graders inputs allocating the ODC from randomly selected images is shown in Fig. 4. It was observed that the distribution could be fitted by a Gaussian function,

$$
G(x)=136.16 \times e^{-\left(x-x_{0}\right)^{2} /\left(2 \sigma^{2}\right)}
$$




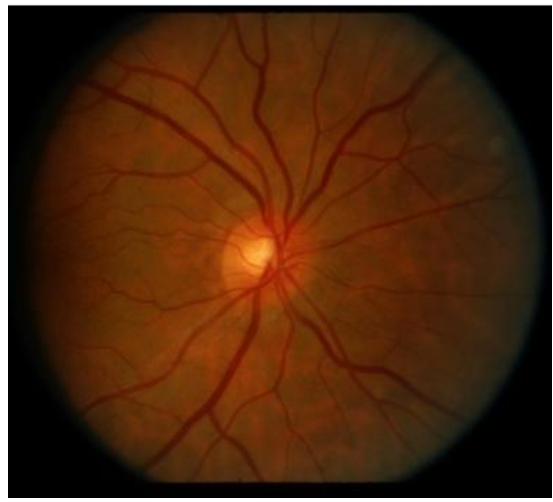

(a)

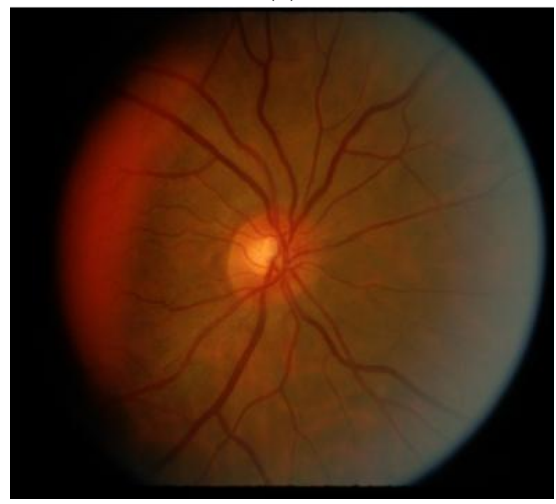

(b)

Fig. 6. Stereo image. (a) Retina image taken from the right to the optical axis. (b) Retina image taken from the left to the optical axis.

where $x_{0}=0.844, \sigma=6.051$.

Using Gaussian distribution (11), 100 Monte Carlo estimates of the optic centre were obtained for each image. Fig. 5 shows three examples of different ROIs generated by this technique.

\section{F. Effect of Angle of Imaging (Reliability Test)}

Ideally, the retina image should be taken with the camera lined with the optical axis of the eye. However, this may not always be the case. Variations in the angle can result in changes in the light intensity distribution and also cause variations in the shadow effects in the image, resulting in poor repeatability of the experiments [31]. The technique proposed in this paper overcomes this shortcoming because $F F D$ is less sensitive to noise as it does not require the image to be binarized. While the gray scale image analysis are also sensitive to noise and variations, we hypothesis that binarization tends to exaggerate this and causes the large variations in the outcome.

To verify the improved repeatability of this technique, 30 stereo pairs of retina images $(n=60)$ were used. Each photo of the two in these pairs was taken using the same camera as described previously, from different photographic angles to obtain a stereo view of the image pairs.

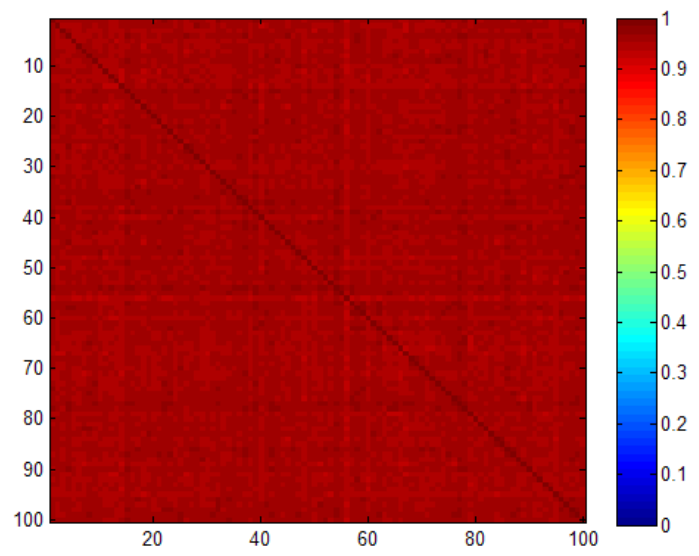

Fig. 7. Heat map shows pair-wise correlation of 380 FFD measurements over 100 different ROIs. The diagonals are correlations of $F F D$ from the same ROI (the mean correlation is 0.93 after removing the diagonals).

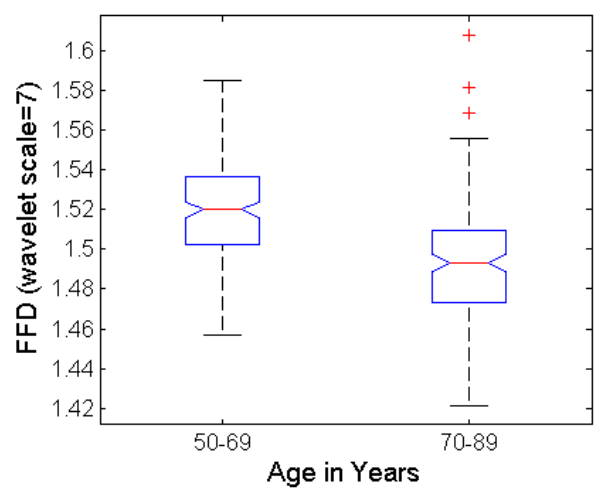

Fig. 8. Fourier Fractal dimension for the two age groups $(\mathrm{n}=380)$.

If the measurements of the images taken from the right and the left angles correlate, the technique can be considered to be insensitive to the relative angle between the camera optical axis and the eye optical axis. Fig. 6(a) and Fig. 6(b) were captured few seconds apart and the angle of imaging differs by approximately 7 degrees. The differences in lighting and resultant shadows are evident from the two images.

\section{G. Validation using Age-based Data}

To test the efficacy of this technique, age-based data were analyzed. The reduction in the complexity of the body has been recognized by [25]. While reduction in $F D$ of the retina scan is expected with aging, most $F D$ analysis of the retina using box-counting approach has been unable to report significant reduction in the $F D$ with aging [13-15]. Experiments were conducted to obtain the $F D$ of the retina images using FFD and the significance of separation of the 2 classes was taken as a measure of the efficacy of this technique.

\section{RESULTS}

Table I shows the correlation (for 100 images) between the known and computed $F F D$ of the noisy image. This table shows that there is a strong linear relationship between the two 
This article has been accepted for publication in a future issue of this journal, but has not been fully edited. Content may change prior to final publication.

and thus validates the robustness of $F F D$ measurement

\begin{tabular}{|l|r|}
\hline \multicolumn{2}{|c|}{ TABLE I } \\
\multicolumn{2}{|c|}{ PEARSON'S CORRELATION COEFFICIENT OF THE } \\
NOISY FFD WITH THE THEORETICAL $F F D$
\end{tabular}

technique against noises.

\begin{tabular}{|c|r|c|c|}
\hline \multicolumn{4}{|c|}{ TABLE II } \\
\hline INTRA-GRADERS REPEATABILITY EXPERIMENT \\
\hline Method & $\begin{array}{c}\text { Num } \\
\text { ber of } \\
\text { Images }\end{array}$ & $\begin{array}{c}\text { Number of } \\
\text { Graders }\end{array}$ & Correlation \\
\hline Box-counting [16] & 60 & $\begin{array}{c}2 \\
\text { graders }\end{array}$ & 0.93 \\
\hline FD7 & 380 & $\begin{array}{l}100 \\
\text { simulated } \\
\text { graders }\end{array}$ & 0.93 \\
\hline
\end{tabular}

\begin{tabular}{|l|c|c|}
\hline \multicolumn{3}{|c|}{ TABLE III } \\
\hline Vessel Segmentation/Enhancement Method & $\begin{array}{c}\text { Method to } \\
\text { Estimate Complexity }\end{array}$ & Correlation \\
\hline $\begin{array}{l}\text { Nonlinear-orthogonal projection segmentation } \\
{[33]}\end{array}$ & Box-counting & $\begin{array}{c}0.57 \text { [Centre for Eye } \\
\text { Research Australia, personal } \\
\text { communication, March 20, } \\
\text { 2010] }\end{array}$ \\
\hline Curvature-based retinal vessel segmentation [17] & Box-counting & $\begin{array}{c}0.48 \text { [Centre for Eye } \\
\text { Research Australia, personal } \\
\text { communication, March 20, } \\
2010]\end{array}$ \\
\hline Gabor Wavelet supervised segmentation [22] & Box-counting & 0.61 \\
\hline Gabor Wavelet enhancement (scale 4) & $F F D$ & 0.65 \\
\hline Gabor Wavelet enhancement (scale 5) & $F F D$ & 0.64 \\
\hline Gabor Wavelet enhancement (scale 6) & $F F D$ & 0.60 \\
\hline Gabor Wavelet enhancement (scale 7) & $\boldsymbol{F F D}$ & $\mathbf{0 . 7 4}$ \\
\hline Gabor Wavelet (scale 8) & $F F D$ & 0.55 \\
\hline
\end{tabular}

\begin{tabular}{|c|c|c|}
\hline \multicolumn{3}{|c|}{$\begin{array}{c}\text { TABLE IV } \\
\text { STATISTICAL SIGNIFICANCE OF RELATIONSHIP OF AGING WITH CHANGES IN } F D\end{array}$} \\
\hline Method & $\begin{array}{l}\text { Pearson's Correlation } \\
\quad \text { Coefficient, } r\end{array}$ & P-Value \\
\hline $\begin{array}{l}\text { Box-counting on skeletonized vessel D0 } \\
\text { [13] }\end{array}$ & -0.2017 & 0.3561 \\
\hline $\begin{array}{l}\text { Box-counting on skeletonized vessel D1 } \\
\text { [13] }\end{array}$ & -0.1270 & 0.5636 \\
\hline Box-counting on skeletonized vessel D2[13] & -0.0582 & 0.7919 \\
\hline Box-counting on vessel tracing [14] & 0.5061 & 0.1356 \\
\hline Box-counting on vessel width [16] & -0.42 & $<0.0001$ \\
\hline$F F D$ & -0.45 & $<0.0001$ \\
\hline
\end{tabular}

The mean (for 380 images) of the Pearson's correlation coefficient between the fractal dimension computed using $F F D$ for the 100 simulated ROI of each of the images are tabulated in Table II. This is a measure of the independence of the outcome from the grader expertise, thereby the measure of the repeatability of the technique. The results show that the mean correlation coefficient (standard deviation) was $0.93(0.005)$ after removing correlations of same elements, when the ROI was based on 15 untrained graders which is the same as correlation achieved when 2 expert graders were identifying the ROI when using box-counting approach.

Fig. 7 shows the heat map of the Pearson's correlation of 380 FD7 measured from 100 ROIs. The diagonals are the same ROI pair-wise correlations. Table II compares FFD with boxcounting technique [16]. Table III is a comparison of the correlation between the $F D$ of the stereo images based on box counting approach and FFD. It indicates that while the outcome of using the box-counting approach was sensitive to the difference between the stereo images having approximately an axial separation of 7 degrees, $F F D$ was much less sensitive. The results also indicate that the best result was obtained when the image was enhanced using Gabor wavelets scale 7.

The FFD box-plot of the two age classes is shown in Fig. 8 . The box-plot demonstrates a measurable reduction in $F D$, with significant difference between the two classes $(p<0.0001)$. This is consistent with findings of reduced complexity associated with aging in other parts of the body [23, 24, 32]. Table IV compares our result with box-counting techniques previously reported in literature [13]. D0, D1, and D2 correspond to box-counting technique measured at different resolutions. 


\section{DisCUSSION AND CONCLUSION}

Automatic vessel segmentation of retina images is essential for computing fractal dimension based on box-counting technique. While excellent vessel segmentation techniques have been developed and reported in literature, [17, 22, 33], these have not been tested on datasets with variations in illumination conditions. When the automated vessel segmentation method was tested on dataset with larger variation, there was a need for manual intervention which is expensive and the outcomes are grader expertise dependent [16].

We have developed and tested a reliable method to measure the fractal dimension of retina images. This includes the preprocessing of the retina image to improve its quality using wavelet transforms, and the use of Fourier Fractal dimension $(F F D)$ to obtain the $F D$ of the image without requiring the binarization of the image. The proposed technique does not require segmentation of the vessels and thus has overcome the earlier shortcoming. This makes it suitable for automated retina image analysis and thus makes it possible for analysis of large data-sets, which has not been possible due to the need for expert graders for manual intervention. It also makes the outcomes more reliable as these are not dependent on the graders' expertise.

The proposed technique has been first validated using synthetic noisy images. The results show a significant correlation between the known and computed noisy FFD (Table I). This confirms the robustness of FFD against noise.

Experiments were also conducted to test the impact of the angle between the camera and the optical axis. Changes in this angle cause variations in the brightness of the image (Fig. 6) and can also change the direction of shadows in the image. There can be a significant impact from these shadows when the image is binarized. Some vessels that are observed in the segmented image from one angle may be missed from another angle. The results of the experiments (Table III) demonstrate that the outcomes are more reliable and less sensitive to the angle between the camera and the optical axis of the eye compared with box-counting method. This is because FFD does not require the segmentation of the image and there is no global threshold. Thus the impact of the angle of the camera, which results in variations in the local conditions in the image, is not large. The results indicate that wavelet transform at scale 7 provides the best results in terms of reliability. This indicates that at this scale, the noise and artifacts get diminished while only the relevant retinal vasculature is highlighted, making it less sensitive to variations in the camera angle.

To compare this technique with box-counting technique, data with known relationship (impact of aging) was studied. While many previous studies using box-counting approach have been inconclusive regarding the relationship of changes in the FD with aging [13-15], the results of experiments conducted using 380 retina images demonstrate that this technique was able to identify the reduction in complexity measured as $F D$ with aging (Fig. 8 and Table IV). Our technique has experimentally validated the expected decrease in $F D$ with aging [23, 24, 32], results being comparable with FD computed based on vessel width which requires high level of manual intervention [16].

The novelty of this technique lies in the use of FFD on grayscale retina images, eliminating the binary segmentation of the vessel network which is prone-to-error whether carried out by human expert or by automated algorithms. The technique is also insensitive to the choice of the optic centre (within the range of average grader, Table II). The other factor that improves the overall performance of this method is the appropriate choice of wavelet scale for matched filters reduces the impact of local differences that happen due to the change in the illumination conditions. This is evident when comparing the employed FFD and the Box-Counting methods, a) the better reliability of $F F D$ with higher correlation of 0.74 , and b) the consistent finding of statistically significant reduction of complexity with aging $(r=-0.45, p<0.0001)$. This technique provides an important basis for further exploring the potential for fractal measurements of retinal vasculature to be used as subclinical markers for systemic vascular conditions such as heart disease, stroke, diabetes and other conditions which deserves further exploration [16].

\section{ACKNOWLEDGMENT}

The authors thank the staff and participants of the BMES for their important contributions.

\section{REFERENCES}

[1] J. J. Wang, P. Mitchell, H. Leung, E. Rochtchina, T. Y. Wong, and R. Klein, "Hypertensive retinal vessel wall signs in a general older population: the Blue Mountains Eye Study," Hypertension, vol. 42, p. 534, 2003

[2] T. Y. Wong, R. Klein, A. R. Sharrett, T. A. Manolio, L. D. Hubbard, E. K. Marino, L. Kuller, G. Burke, R. P. Tracy, J. F. Polak, J. S. Gottdiener, and D. S. Siscovick, "The prevalence and risk factors of retinal microvascular abnormalities in older persons: The cardiovascular health study," Ophthalmology, vol. 110, pp. 658-666, 2003.

[3] T. Y. Wong, M. D. Knudtson, R. Klein, B. E. K. Klein, S. M. Meuer, and L. D. Hubbard, "Computer-assisted measurement of retinal vessel diameters in the Beaver Dam Eye Study: Methodology, correlation between eyes, and effect of refractive errors," Ophthalmology, vol. 111, pp. 1183-1190, 2004.

[4] T. Y. Wong and P. Mitchell, "The eye in hypertension," Lancet, vol. 369, pp. 425-435, 2007.

[5] G. Liew, J. Wang, P. Mitchell, and T. Wong, "Retinal vascular imaging: a new tool in microvascular disease research," Circulation: Cardiovascular Imaging, vol. 1, p. 156, 2008.

[6] T. Y. Wong, "Is retinal photography useful in the measurement of stroke risk?," Lancet Neurology, vol. 3, pp. 179-183, 2004.

[7] T. Y. Wong, R. Klein, D. J. Couper, L. S. Cooper, E. Shahar, L. D. Hubbard, M. R. Wofford, and A. R. Sharrett, "Retinal microvascular abnormalities and incident stroke: the Atherosclerosis Risk in Communities Study," The Lancet, vol. 358, pp. 1134-1140, 2001. 
[8] T. Y. Wong, R. Klein, A. R. Sharrett, B. B. Duncan, D. J. Couper, J. M. Tielsch, B. E. K. Klein, and L. D. Hubbard, "Retinal arteriolar narrowing and risk of coronary heart disease in men and women: the Atherosclerosis Risk in Communities Study," Jama, vol. 287, p. 1153, 2002.

[9] T. Y. Wong, R. Klein, A. R. Sharrett, M. I. Schmidt, J. S. Pankow, D. J. Couper, B. E. K. Klein, L. D. Hubbard, and B. B. Duncan, "Retinal arteriolar narrowing and risk of diabetes mellitus in middle-aged persons," Jama, vol. 287, p. 2528, 2002.

[10] A. D. Hughes, E. Martinez-Perez, A. S. Jabbar, A. Hassan, N. W. Witt, P. D. Mistry, N. Chapman, A. V. Stanton, G. Beevers, R. Pedrinelli, K. H. Parker, and S. A. M. Thom, "Quantification of topological changes in retinal vascular architecture in essential and malignant hypertension," Journal of Hypertension, vol. 24, pp. 889-894, 2006.

[11] N. Witt, T. Y. Wong, A. D. Hughes, N. Chaturvedi, B. E. Klein, R. Evans, M. McNamara, S. A. McG Thom, and R. Klein, "Abnormalities of retinal microvascular structure and risk of mortality from ischemic heart disease and stroke," Hypertension, vol. 47, pp. 975-981, 2006.

[12] M. B. M. Mendonça, C. A. A. Garcia, R. A. Nogueira, M. A. F. Gomes, M. M. Valença, and F. Oréfice, "Fractal analysis of retinal vascular tree: segmentation and estimation methods," Arquivos Brasileiros de Oftalmologia, vol. 70, pp. 413-422, 2007.

[13] G. Landini, G. P. Misson, and P. I. Murray, "Fractal analysis of the normal human retinal fluorescein angiogram," Current eye research, vol. 12, pp. 23-27, 1993.

[14] B. R. Masters, "Fractal analysis of the vascular tree in the human retina," in Annual Review of Biomedical Engineering. vol. 6, 2004, pp. 427-452.

[15] F. Family, B. R. Masters, and D. E. Platt, "Fractal pattern formation in human retinal vessels," Physica D: Nonlinear Phenomena, vol. 38, pp. 98-103, 1989.

[16] G. Liew, J. J. Wang, N. Cheung, Y. P. Zhang, W. Hsu, M. L. Lee, P. Mitchell, G. Tikellis, B. Taylor, and T. Y. Wong, "The Retinal Vasculature as a Fractal: Methodology, Reliability, and Relationship to Blood Pressure," Ophthalmology, vol. 115, pp. 1951-1956.e1, 2008.

[17] S. Garg, J. Sivaswamy, and S. Chandra, "Unsupervised curvature-based retinal vessel segmentation," in 4th IEEE International Symposium on Biomedical Imaging: From Nano to Macro, 2007. ISBI 2007, 2007, pp. 344-347.

[18] J. C. Russ, Fractal surfaces: Plenum Pub Corp, 1994.

[19] J. A. Loaiza, "Experimental demonstration of the fractal nature of unstable-resonator modes." vol. Ph.D. Leiden: Leiden University, 2005.

[20] E. Gaudio, S. Chaberek, A. Montella, L. Pannarale, S. Morini, G. Novelli, F. Borghese, D. Conte, and K. Ostrowski, "Fractal and Fourier analysis of the hepatic sinusoidal network in normal and cirrhotic rat liver," Journal of Anatomy, vol. 207, p. 107, 2005.
[21] L. Gang, O. Chutatape, and S. M. Krishnan, "Detection and measurement of retinal vessels in fundus images using amplitude modified secondorder Gaussian filter," IEEE Transactions on Biomedical Engineering, vol. 49, pp. 168-172, 2002.

[22] J. V. B. Soares, J. J. G. Leandro, R. M. Cesar Jr, H. F. Jelinek, and M. J. Cree, "Retinal vessel segmentation using the 2-D Gabor wavelet and supervised classification," IEEE Transactions on Medical Imaging, vol. 25, pp. 1214-1222, 2006

[23] A. L. Goldberger, L. A. N. Amaral, J. M. Hausdorff, P. C. Ivanov, C. K. Peng, and H. E. Stanley, "Fractal dynamics in physiology: alterations with disease and aging," Proceedings of the National Academy of Sciences, vol. 99, p. 2466, 2002.

[24] A. L. Goldberger, C. K. Peng, and L. A. Lipsitz, "What is physiologic complexity and how does it change with aging and disease?," Neurobiology of aging, vol. 23, pp. 23-26, 2002.

[25] L. A. Lipsitz and A. L. Goldberger, "Loss of 'complexity' and aging: Potential applications of fractals and chaos theory to senescence," Journal of the American Medical Association, vol. 267, pp. 1806-1809, 1992.

[26] P. Mitchell, W. Smith, K. Attebo, and J. J. Wang, "Prevalence of agerelated maculopathy in Australia. The Blue Mountains Eye Study," Ophthalmology, vol. 102, p. 1450, 1995.

[27] A. Osareh and B. Shadgar, "Automatic blood vessel segmentation in color images of retina," Iranian Journal of Science and Technology, Transaction B: Engineering, vol. 33, pp. 191-206, 2009.

[28] R. M. Rangayyan, F. Oloumi, P. Eshghzadeh-Zanjani, and F. J. Ayres, "Detection of blood vessels in the retina using gabor filters," in Canadian Conference on Electrical and Computer Engineering, 2007, pp. 717-720.

[29] R. M. Rangayyan, Biomedical image analysis: CRC, 2005.

[30] J. J. Lennon, "Red-shifts and red herrings in geographical ecology," Ecography, vol. 23, pp. 101-113, 2000.

[31] M. Ortega, M. G. Penedo, J. Rouco, N. Barreira, and M. J. Carreira, "Retinal verification using a feature points-based biometric pattern," EURASIP Journal on Advances in Signal Processing, vol. 2009, p. 2, 2009.

[32] L. A. Lipsitz and A. L. Goldberger, "Loss of complexity and aging," Jama, vol. 267, pp. 1806-1809, 1992.

[33] Y. Zhang, W. Hsu, and M. L. Lee, "Detection of Retinal Blood Vessels Based on Nonlinear Projections," Journal of Signal Processing Systems, vol. 55, pp. 103-112, 2009. 